\title{
Sobrepeso e obesidade em adolescentes de escolas
}

\section{públicas}

\author{
Recebido em 15/08/2012 \\ Aprovado em: 16/05/2013
}

\author{
Eline Saraiva Silveira Araújo \\ Maria Vilani Cavalcante Guedes ${ }^{2}$ \\ Paulo César Almeida ${ }^{3}$
}

Resumo: Objetivou-se verificar a frequência de obesidade e sobrepeso em adolescentes de escolas públicas por meio da idade e antropometria. Estudo transversal com 660 alunos, com idade de 12 a 19 anos, cuja coleta ocorreu em março de 2012. Dos adolescentes, 344 (52,1\%) eram meninas. A média de idade dos escolares situou-se entre $16 \pm 1,7$ ano, com amplitude de variação de 12 a 19 anos. A maioria, 541 (82\%), apresentou IMC saudável, porém $86(13 \%)$ foram considerados de risco de sobrepeso ou sobrepeso. Urge refletir e explorar a problemática, mediante desenvolvimento de atividades de prevenção e ações interventivas nos casos encontrados.

Descritores: Adolescente, Antropometria, Sobrepeso, Obesidade

\section{Overweight and obesity among adolescents in public schools}

Abstract: We aimed to verify the frequency of obesity and overweight in adolescents from public schools through age and anthropometry. A crosssectional study with 660 students, aged from 12-19 years, whose data collection happened in March 2012. From the adolescents, 344 (52.1\%) were female. The average age of the students was $16 \pm 1.7$ year, ranging from 12 to 19 years. The majority, 541 ( $82 \%$ ), presented healthy BMI, however 86 (13\%) were considered at risk for overweight or overweight. There is urgent need to reflect and explore the issue by developing preventive activities and interventional actions in the cases found.

Descriptors: adolescent. Anthropometry, Overweight, obesity

\section{Sobrepeso y obesidad en adolescentes de escuelas públicas.}

Resumen: El objetivo fue verificar la frecuencia de obesidad y sobrepeso en adolescentes de escuelas públicas por edad y antropometría. Estudio transversal, con 660 alumnos, de 12 a 19 años, en marzo de 2012. De los adolescentes, 344 (52,1\%) eran niñas. El promedio de edad estuvo entre $16 \pm$ 1,7 año, con rango de variación entre 12 y 19 años. La mayoría, 541 (82\%), presentó IMC saludable, pero 86 (13\%) se consideraron en riesgo de sobrepeso o sobrepeso. Es preciso reflexionar y estudiar la problemática a través del desarrollo de actividades de prevención y acciones de intervención en los casos encontrados.

Descriptores: adolescente, Antropometría, Sobrepeso, Obesidad

\section{INTRODUÇÃO}

$\mathrm{P}$ or adolescência compreende-se a etapa entre infância e fase adulta, período no qual acontecem várias mudanças, psicológicas, hormonais e antropométricas. É a segunda década da vida, ocorrendo entre 10 e 19 anos, marcada por complexo processo de crescimento e desenvolvimento biopsicossocial ${ }^{(1)}$.

O crescente índice de obesidade observado nos últimos anos tem assumido caráter epidêmico, tornando-se problema de saúde. Estudo recente, aponta transição epidemiológica, de contexto de desnutrição para cenário de sobrepeso.

A obesidade na adolescência está associada a doenças crônicas na vida adulta como hipertensão, dislipidemias e diabetes ${ }^{(2,3,4)}$. Determinados fatores parecem exercer influências, tais como: diversidades culturais, variações climáticas, fatores genéticos e diferenças socioeconômicas ${ }^{(5,6)}$. Ademais, as condições de moradia e higiene podem exercer impactos no estilo de vida e na saúde das pessoas ${ }^{(6,7)}$.

Na mencionada fase, a obesidade aumenta para $80 \%$ a possibilidade de se prolongar na vida adulta. De acordo com estimativas, no futuro haverá mais adultos que, além de obesos, apresentarão doenças com raízes na infância, como a doença cardiovascular ${ }^{(8)}$. Diante das evidências, enfatiza-se a importância de identificar e tratar de modo efetivo o sobrepeso em adolescentes.

Neste âmbito, recomenda-se o uso da antropometria, técnica de fácil aplicabilidade, que por ser um método prático, com baixo custo, não invasivo e de aceitação favorável pela população, fornece estimativas da prevalência e gravidade das alterações nutricionais.

Um destes métodos é o Índice de Massa Corporal (IMC), amplamente utilizado como válido para verificação de indicadores nutricionais e de condições de vida da população, o qual é usado como parâmetro eficaz para expressar a gordura corporal em excesso ${ }^{(9,10)}$.

$\mathrm{Na}$ população inserida no ambiente escolar, o acompanhamento da situação nutricional é essencial para análise da saúde, tornando-se oportunidade ímpar para obtenção de medidas objetivas da evolução das condições de vida da referida população.

O monitoramento da prevalência de excesso de peso em adolescentes permite verificar tendências ao longo do tempo, além de identificar subgrupos desta população mais expostos a esse problema ${ }^{(9)}$. Neste contexto, o acompanhamento do 
crescimento e desenvolvimento desde a infância torna-se essencial na enfermagem, para que a detecção e o tratamento da obesidade sejam executados de forma precoce e eficiente. Consoante refletem estudos, as atividades de educação em saúde devem utilizar estratégias metodológicas mais inclusivas, direcionadas e lúdicas ${ }^{(11)}$.

Para atingir esse público, são desenvolvidas atividades de integração e articulação permanente entre a Estratégia Saúde da Família (ESF) e a educação básica. Instituído em 2007 pelo governo federal, o Programa Saúde na Escola (PSE) volta-se a crianças, adolescentes e jovens, estudantes de escolas públicas brasileiras ${ }^{(12)}$. Assim, em 2012, ocorreu a Semana de Mobilização da Saúde na Escola(13), sobre prevenção da obesidade, trabalhada pela escola em parceria com a ESF, com foco nos escolares e famílias ${ }^{(1)}$.

Diante dessa realidade, este estudo objetivou verificar a frequência de obesidade e sobrepeso em adolescentes de escolas públicas por meio da idade e antropometria.

\section{MÉTODOS}

Neste estudo adotou-se abordagem de natureza quantitativa, de caráter descritivo exploratório, do tipo transversal, com alunos do 60 ao 90 ano do ensino fundamental e do 10 ao 30 ano do ensino médio de duas escolas públicas, localizadas em Fortaleza, Ceará. A coleta deu-se em março de 2012, nos turnos manhã, tarde e noite.

Para a verificação do peso corporal trabalhou-se com balança digital portátil com capacidade de 100 kg e com precisão de $100 \mathrm{~g}$, colocada em superfície plana. Os escolares foram pesados descalços, com o mínimo de roupa possível, corpo ereto no centro da balança, braços estendidos ao longo do corpo e sem movimento. Para medição da altura, o instrumento foi um antropômetro, com subdivisão 0,5 $\mathrm{cm}$, verificado com esquadro sobre a cabeça dos escolares, descalços, pés unidos e paralelos, em posição ereta e olhando para a frente. Para informação sobre idade, consultou-se o arquivo da escola.

Calcularam-se para o IMC e idade as medidas médias, o desvio padrão e o erro padrão da média (EPM). As categorias de IMC foram realizadas segundo os percentis: I) Se < P5 - baixo peso; II) entre P5 e P85 - peso saudável; III) entre P85 e P95 - risco de sobrepeso e IV) se $\geq$ P95 - sobrepeso. Analisou-se a associação entre as categorias de IMC e as variáveis sociodemográficas por meio do teste de $\mathrm{x}^{2}$. Verificou-se a normalidade do IMC pelo teste de Kolmogorov-
Smirnov e a igualdade de variâncias pelo teste de Levine. Enquanto as médias de IMC foram comparadas pelo teste t de Student e pelo teste F de Snedecor, as comparações múltiplas foram feitas pelo teste de Tukey. Foram consideradas, estatisticamente, as análises inferenciais com $\mathrm{p}<0,05$. Os dados foram processados pelo EPI-INFO.

Como determinado, este estudo foi aprovado pelo Comitê de Ética em Pesquisa da instituição lócus da pesquisa, conforme parecer $070716 / 10$, em consonância com a Resolução 196/96, do Conselho Nacional de Saúde. O Termo de Consentimento foi assinado por eles ou por seus responsáveis, para os menores de 18 anos.

\section{RESULTADOS}

Expõem-se a seguir, na Tabela 1, os dados referentes às características demográficas e ao IMC dos adolescentes pesquisados.

Tabela 1 - Distribuição do número de adolescentes, segundo características demográficas. Fortaleza-CE, Brasil, 2012

\begin{tabular}{|c|c|c|c|}
\hline Características & No & $\%$ & \\
\hline \multicolumn{4}{|l|}{ Série } \\
\hline $6^{a}$ a $9^{a *}$ & 387 & 58,7 & \\
\hline 1 a $a 3^{a * *}$ & 273 & 41,3 & \\
\hline \multicolumn{4}{|l|}{ Idade } \\
\hline $12-14$ & 181 & 27,4 & \multirow{3}{*}{$\begin{array}{c}\text { Média } \pm \text { DP } \\
16 \pm 1,7 \\
\text { anos }\end{array}$} \\
\hline $15-16$ & 244 & 37,0 & \\
\hline $17-19$ & 235 & 35,6 & \\
\hline \multicolumn{4}{|l|}{ Sexo } \\
\hline Masculino & 316 & 47,9 & \\
\hline Feminino & 344 & 52,1 & \\
\hline \multicolumn{4}{|l|}{ IMC (percentil)' } \\
\hline Baixo peso & 33 & 5,0 & \\
\hline Saudável & 541 & 82,0 & \\
\hline $\begin{array}{l}\text { Risco de } \\
\text { sobrepeso }\end{array}$ & 63 & 9,5 & \\
\hline Sobrepeso & 23 & 3,5 & \\
\hline
\end{tabular}

*Ensino fundamental; **Ensino médio.

'IMC percentil: baseado nos percentis e diferentemente para adolescentes do sexo masculino e feminino.

Como mostra a Tabela 1, as menores séries de estudo (6a a 9a) apresentaram maior percentual de alunos, 387 (58,7\%). A maioria dos adolescentes era menina, 344(52,1\%). Quanto à média de idade, variou de $16 \pm 1$,7ano, com amplitude de variação de 12 a 19 anos. E foi igual em ambos os sexos ( $p>0,05)$. A maior parte, 541(82\%), denotou IMC saudável, contudo $86(13 \%)$ foram considerados de risco de sobrepeso ou com sobrepeso.

Na Tabela 2, constam as análises de associação entre IMC e as características dos adolescentes. 
Tabela 2 - Distribuição do número de adolescentes, segundo o IMC e as características. Fortaleza-CE. Brasil, 2012

\begin{tabular}{|c|c|c|c|c|c|c|c|c|c|}
\hline IMC & & & & & Risco & repeso & & repe & \\
\hline Características & No & $\%$ & No & $\%$ & No & $\%$ & No & $\%$ & $\mathbf{P}$ \\
\hline Série & & & & & & & & & 0.006 \\
\hline $6^{a}$ a $9^{a}$ & 25 & 6,5 & 323 & 83,4 & 26 & 6,7 & 13 & 3,4 & \\
\hline $1^{\mathrm{a}}$ a $3^{\mathrm{a}}$ & 8 & 2,9 & 218 & 79,9 & 37 & 13,5 & 10 & 3,7 & \\
\hline Sexo & & & & & & & & & 0,959 \\
\hline Masculino & 15 & 4,7 & 260 & 82,3 & 31 & 9,8 & 10 & 3,2 & \\
\hline Feminino & 18 & 5,2 & 281 & 81,7 & 32 & 9,3 & 13 & 3,8 & \\
\hline Faixa etária (ano) & & & & & & & & & 0,005 \\
\hline $12-14$ & 18 & 9,9 & 148 & 81,8 & 10 & 5,5 & 5 & 2,8 & \\
\hline $15-16$ & 8 & 3,3 & 203 & 83,2 & 23 & 9,4 & 10 & 4,1 & \\
\hline $17-19$ & 7 & 3,0 & 190 & 80,8 & 30 & 12,8 & 8 & 3,4 & \\
\hline Sexo Masculino (Idade) & & & & & & & & & 0,092 \\
\hline $12-14$ & 8 & 10,8 & 58 & 78,4 & 6 & 8,1 & 2 & 2,7 & \\
\hline $15-16$ & 4 & 3,3 & 103 & 84,4 & 11 & 9,0 & 4 & 3,3 & \\
\hline $17-19$ & 3 & 2,5 & 99 & 82,5 & 14 & 11,7 & 4 & 3,3 & \\
\hline Sexo Feminino (Idade) & & & & & & & & & 0,016 \\
\hline $12-14$ & 10 & 9,4 & 90 & 84,1 & 4 & 3,7 & 3 & 2,8 & \\
\hline $15-16$ & 4 & 3,3 & 100 & 82 & 12 & 9,8 & 6 & 4,9 & \\
\hline $17-19$ & 3 & 3,5 & 91 & 79,1 & 16 & 13,9 & 4 & 3,5 & \\
\hline Sexo Masculino (Série) & & & & & & & & & 0,027 \\
\hline $6^{a}$ a $9^{a}$ & 13 & 7,3 & 148 & 82,7 & 13 & 7,3 & 5 & 2,7 & \\
\hline $1^{\text {a }}$ a $3^{a}$ & 2 & 1,5 & 112 & 81,8 & 18 & 13,1 & 5 & 3,6 & \\
\hline Sexo Feminino (Série) & & & & & & & & & 0,095 \\
\hline $6^{a}$ a $9^{a}$ & 12 & 5,8 & 175 & 84,5 & 13 & 6,3 & 8 & 3,4 & \\
\hline $1^{a} a 3^{a}$ & 6 & 4,4 & 106 & 77,9 & 19 & 14 & 5 & 3,7 & \\
\hline
\end{tabular}

$\overline{p d e} x^{2}$

Conforme se observa na Tabela 2, os adolescentes das faixas de escolaridade mais elevadas apresentaram maior porcentagem de sobrepeso e baixo peso, enquanto nas menores faixas ocorreu o inverso: maior porcentagem para baixo peso $(p=0,006)$. Não se encontrou associação entre IMC e sexo $(p=0,959)$. Com relação à idade, aqueles da faixa de 17 a 19 anos evidenciaram maior percentual, 38(16,2\%), de risco de sobrepeso ou sobrepeso, enquanto $15(8,3 \%)$ dos menores de 14 anos estavam nessas duas faixas de IMC. Quanto ao baixo peso, 3,0\% dos maiores de 16 anos estavam nessa faixa, enquanto $13,2 \%$ na faixa de 12 a 16 anos apresentaram baixo peso $(p=0,005)$.

Ao se ajustar o IMC segundo o sexo, não se identificou associação entre IMC e faixa etária para o sexo masculino $(p=0,092)$. Contudo, para o sexo feminino, aquelas com maior faixa de idade mostraram maior percentual com risco de sobrepeso ou sobrepeso $(17,4 \%)$ do que as de menor faixa etária $(6,5 \%)(p=0,016)$. Ainda como observado, $16,7 \%$ dos adolescentes do sexo masculino e de maior nível de escolaridade estiveram na faixa de IMC de risco de sobrepeso ou sobrepeso, e 10,0\% daqueles de menor nível se situaram nessas duas faixas $(p=0,027)$. No tocante ao sexo feminino, não se encontrou associação entre IMC e faixa de escolaridade $(p=0,095)$.

As comparações das médias de IMC, segundo as variáveis faixa etária, sexo e escolaridade, estão expostas na Tabela 3.
Tabela 3 - Comparação das médias de IMC, segundo as variáveis sociodemográficas. Fortaleza-CE, Brasil, 2012

\begin{tabular}{|c|c|c|}
\hline Variável & Média $\pm E P M$ & $\mathbf{p}$ \\
\hline \multicolumn{2}{|c|}{ Sexo } & $>0,05^{1}$ \\
\hline Masculino & $16,4 \pm 0,09$ & \\
\hline Feminino & $16,1 \pm 0,09$ & \\
\hline \multicolumn{2}{|c|}{ Faixa etária } & $0,002^{2}$ \\
\hline $12-14$ & $20,7^{a} \pm 0,27$ & \\
\hline $15-16$ & $21,4^{\mathrm{a}, \mathrm{b}} \pm 0,24$ & \\
\hline $17-19$ & $21,4 b \pm 0,24$ & \\
\hline \multicolumn{2}{|c|}{ Série } & $<0,0001^{1}$ \\
\hline $6^{a}$ a $9^{a}$ & $20,9 \pm 0,18$ & \\
\hline $1^{\text {a }}$ a $3^{\mathrm{a}}$ & $22,0 \pm 0,23$ & \\
\hline \multicolumn{2}{|c|}{ Sexo masculino } & $0,0112^{2}$ \\
\hline $12-14$ & $20,4^{\mathrm{a}} \pm 0,42$ & 5,0 \\
\hline $15-16$ & $20,7^{\mathrm{a}} \pm 0,30$ & 82,0 \\
\hline $17-19$ & $21,7^{\mathrm{a}, \mathrm{b}} \pm 0,19$ & 9,5 \\
\hline \multicolumn{2}{|c|}{ Sexo feminino } & $0,018^{2}$ \\
\hline $12-14$ & $20,8 a \pm 0,35$ & \\
\hline $15-16$ & $22,1 b \pm 0,37$ & \\
\hline $17-19$ & $22,2 b \pm 0,36$ & \\
\hline
\end{tabular}


1)teste t de Student; 2)teste ANOVA e test post hoc de Tukey; letras iguais, médias iguais; letras diferentes, médias diferentes; EPM = erro padrão da média.

Como mostram os dados, as médias de IMC dos adolescentes de ambos os sexos foram iguais $(p>0,05)$, mas os escolares de menor faixa etária obtiveram menor IMC do que as médias das duas maiores faixas etárias $(p=0,002)$. Ao se ajustar para sexo, segundo se observou, nos meninos, as médias de IMC diferiram da primeira faixa para a última faixa etária $(p=0,017)$, enquanto para as meninas a média de IMC da faixa de 12 a 14 anos diferiu das duas outras faixas de 15-16 $(p=0,036)$ e de 17-19 anos $(p=0,035)$. Os adolescentes de menor faixa de escolaridade apresentaram menor média de IMC $(p<0,0001)$.

\section{DISCUSSÃO}

A média de idade dos participantes estudados foi de 16 anos, com desvio padrão de 1,7 ano, e predomínio na faixa etária de 15 a 16 anos, 244(37\%). Esses resultados corroboram achado de outro estudo, cuja média de idade foi de 16,38 e 16,23 , respectivamente, para o sexo masculino e o feminino ${ }^{(9)}$.

Dos adolescentes avaliados, 344 (52,1\%) eram do sexo feminino. Este dado corrobora estudo semelhante, no qual $59,31 \%$ eram do sexo feminino ${ }^{(3)}$; corrobora, também, outro estudo, $61,5 \%{ }^{(9)}$. Evidencia-se, portanto, a maior presença deste sexo nas avaliações realizadas em escolares. Com relação à série em curso, o sobrepeso esteve presente na faixa de escolaridade maior, enquanto o baixo peso foi maior nas menores faixas escolares.

Para determinação dos índices nutricionais em adolescentes, necessita-se de avaliação antropométrica, bem como de mensurações precisas e rotineiras das medidas corporais. A avaliação do sobrepeso em adolescentes é mais complexa do que em outros grupos etários em virtude da variação no processo de crescimento e desenvolvimento durante a puberdade. Assim, torna-se mister estabelecer critério antropométrico.

Quanto aos IMC encontrados no tocante ao sexo, não se identificaram significâncias estatísticas quando comparados os sexos, cujo risco de sobrepeso e sobrepeso foi observado em $41(13,0 \%)$, no sexo masculino, e em 45 (13,1\%), no feminino. Em outra pesquisa, verificou-se a prevalência de $33 \%$ dos alunos com risco de sobrepeso e sobrepeso, de acordo com a classificação do IMC, sem diferença entre os sexos ${ }^{(1)}$.
No inerente à significância encontrada no sobrepeso do sexo feminino, em todas as faixas de idade, houve maior prevalência com relação ao sexo masculino. Confirma, portanto, estudo que mostrou valores mais elevados no gênero feminino em todas as idades quando comparado ao sexo masculino(10).

Os critérios abordados no estudo utilizaram o IMC como dado antropométrico para classificação de sobrepeso. Este, apesar de possuir correlação favorável com a mensuração de adiposidade em adolescentes, não reflete, com precisão, as mudanças na composição corporal que ocorrem nessa faixa etária, as quais são distintas entre os sexos. Contudo, devido às dificuldades de se utilizar métodos mais sofisticados para medir a gordura corporal, o IMC continua sendo opção viável, de fácil determinação, replicação e alta confiabilidade, não demandando treinamento específico, nem instrumental dispendioso(3).

\section{CONCLUSÃO}

Os resultados obtidos aduziram o aumento de risco de sobrepeso nos adolescentes analisados, a exemplo do que tem sido apontado e discutido pela literatura, a sugerir adoção de educação permanente com escolares. Ademais, o aumento da idade e escolaridade revelou maior frequência de risco de sobrepeso e sobrepeso, não colocando em maior evidência o fator sexo.

Com o incremento da obesidade observado em vários países, entre estes, o Brasil, tornam-se, ainda, mais críticas as avaliações de medidas antropométricas. Urge, pois, refletir e explorar a problemática da obesidade nos adolescentes. Logo, são necessárias ações interventivas nos casos encontrados, como também atividades de prevenção, na busca por melhor qualidade de vida.

Ressalta-se a importância dos enfermeiros da ESF por terem mais oportunidades de lidar com esta clientela no desenvolvimento de diversas ações de forma contínua e permanente. Nestas ações é preciso envolver não só alunos, funcionários e pais para detecção de problemas de saúde, mas também atividades de promoção da saúde e prevenção de agravos.

A despeito das dificuldades e limitações, espera-se que o PSE seja implementado de forma permanente com adolescentes escolares, com intervenções eficazes, na busca por melhores hábitos alimentares. 


\section{Referências}

1. Ministério da Saúde (BR). Marco legal: saúde, um direito de adolescentes. Brasília: Ministério da Saúde; 2005.

2. Pierine DT, Carrascosa APM, Fornazari AC, Watanabe MT, Catalani MCT, Fukuju MM, et al. Composição corporal, atividade física e consumo alimentar de alunos do ensino fundamental e médio. Motriz. 2006; 12(2):113-24.

3.Damasceno MMC, Lopes MVO, Oliveira DD, Nogueira NP, Siqueira IA, Macêdo SF. Sobrepeso em adolescentes de escolas públicas: desempenho de três critérios diagnósticos. Acta Paul Enferm. 2009; 22(2):198-204.

4. Cobayashi F, Oliveira FLC, Escrivão MAMS, Silveira D, Taddei JAAC. Obesidade e fatores de risco cardiovascular em adolescentes de escolas públicas. Arq Bras Cardiol. 2010; 95(2):200-6.

5. Marbá RF, Teixeira HM, Barata NJF, Azevedo NC, Pinto RF. Perfil do crescimento físico dos escolares da rede particular de ensino de Araguaína-TO. Fiep Bul [Internet]. 2010; 80(esp). Disponível em: http: www.fiepbulletin.net/index.php/fiepbulletin/article/ view/2117

6. Leão DO, Barbosa LCA, Mendonça MC, Krebs RJ, Pinto RF. Perfil de crescimento, estado nutricional e avaliação motora dos escolares da região ribeirinha do município de Igarapé Miri, um estudo de caso. Fiep Bul [Internet]. 2010; 80(esp). Disponível em: http: www. fiepbulletin.net/index.php/fiepbulletin/article/view/1682

7. Mendonça MC, Figueiredo RM, Leão DO, Santana MF, Chaves LGS. Avaliação do crescimento físico e estado nutricional dos escolares da unidade de educação infantil "Cremação" -Belém, Pará, Brasil Um estudo de caso. Fiep Bul [Internet]. 2011; 81(esp). Disponível em: http: fiepbulletin.net/index.php/fiepbulletin/article/view/347 8. Marchi-Alves LM, Yagui CM, Rodrigues CS, Mazzo A, Rangel EML, Girão FB. Obesidade infantil ontem e hoje: importância da avaliação antropométrica pelo enfermeiro. Esc Anna Nery. 2011; 15(2):238-44.

9. Romanzini M, Reichert FF, Ronque ERV, Lopes AS, Petroski EL. Determinação do estado nutricional de adolescentes por meio de medidas referidas de peso e estatura: um estudo de validação. Rev Bras Ativ Fís Saúde. 2011; 16(1):31-6.

10. França MAR, Abuquerque Jr RLC, Melo CM. Baixo peso sobrepeso em adolescentes: uma proposta de curva de referência para um estado do Nordeste brasileiro. Fiep Bul [Internet]. 2011; 81(esp). Disponível em: http: //fiepbulletin.net/index.php/ fiepbulletin/article/view/321.

11. Silveira RE, Santos AS. Contextos de vulnerabilidade entre adolescentes do ensino fundamental de Uberaba/MG. Enfermagem em Foco. 2012; 3(4):182-185.

12. Ministério da Saúde (BR). Decreto n. 6.286, de 5 de dezembro de 2007: institui o Programa Saúde na Escola - PSE. Diário Oficial da União, Brasília (DF) 2007 dez 6; Seção 1:4.

13. Ministério da Saúde (BR). Portaria n. 357/GM, de 01 de março de 2012. Semana Anual de Mobilização Saúde na Escola. Diário Oficial da União, Brasília (DF) 2012 mar 21; Seção 2:55. 